\title{
Dynamic Control of Plasmonic Localization by Inverse Optimization of Spatial Phase Modulation
}

\author{
Doksoo Lee ${ }^{\dagger}$, Shizhou Jiang ${ }^{\dagger}$, Oluwaseyi Balogun and Wei Chen* \\ Department of Mechanical Engineering, Northwestern University, Evanston, Illinois 60208, United States \\ ${ }^{\dagger}$ These authors contributed equally to this work.
}

\section{S1. Far-field optics and influence of diffraction}

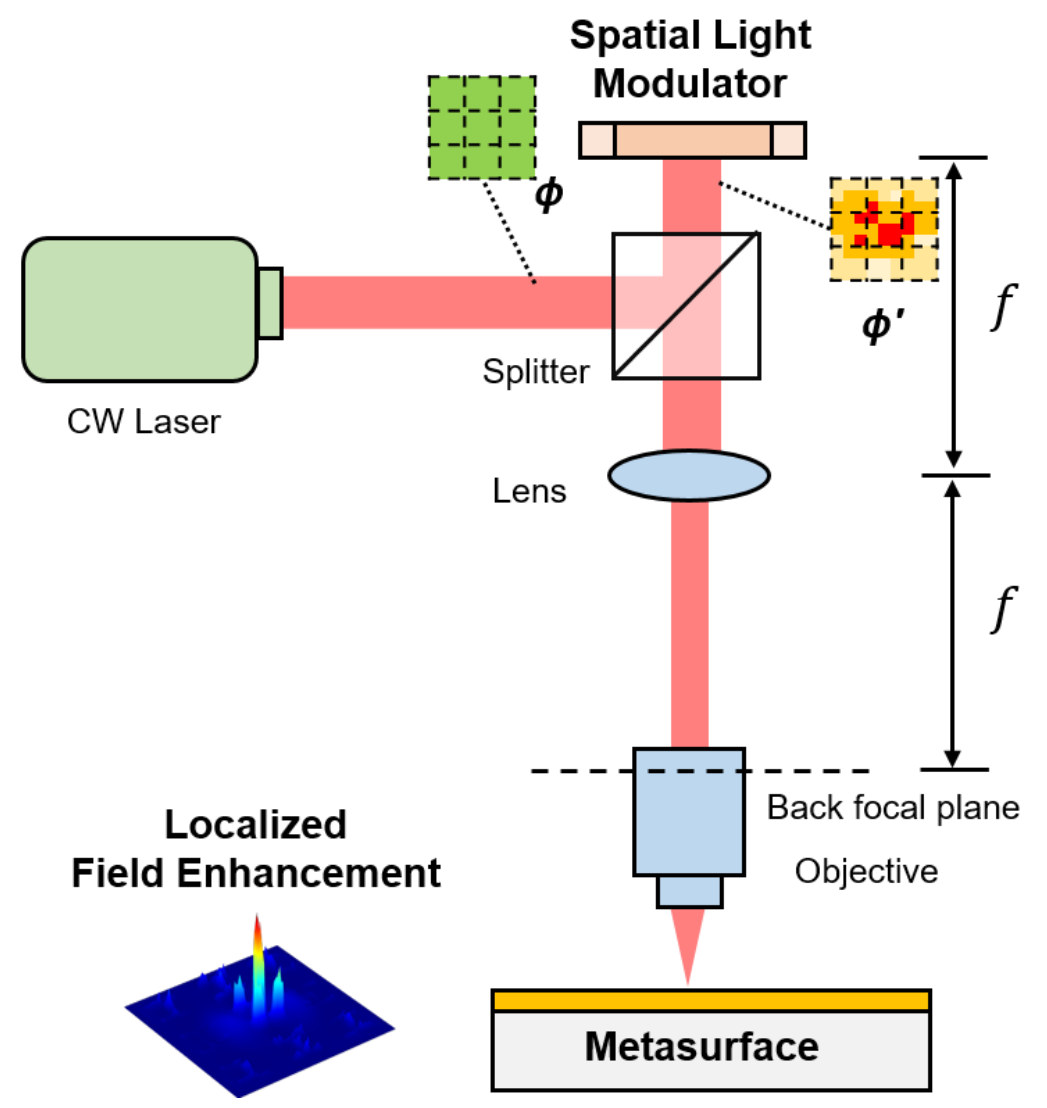

Figure S1. Far-field optics to image incident wavefield optimized by SPM to the metasurface.

One of the advantages of SPM is that the optimized phase distribution can be generated by commercial spatial light modulators (SLMs). The wavefield presented in this paper is defined at the plane of SLM and can be imaged to the metasurface by the imaging system shown in Figure S1 with a demagnification of $M$. The diffraction of the imaging is calculated by considering the objective as a low-pass spatial frequency filter with cutting-off frequency of NA $\cdot k_{0}$ where NA is the numerical aperture of the objective and $k_{0}$ is the free-space wavevector. ${ }^{1}$ 


\section{S2. On the choice of harmonics in the phase representation}

a

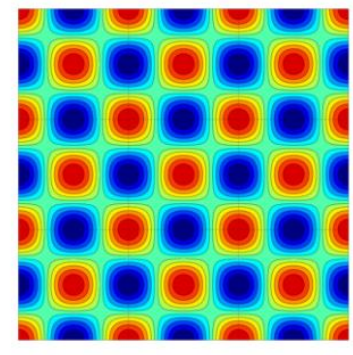

$j=0.5$ b

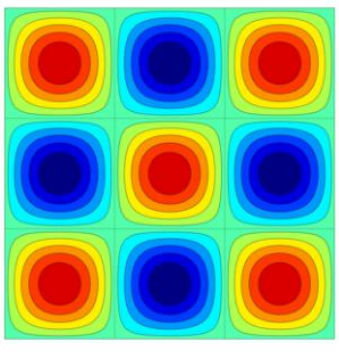

$j=1$

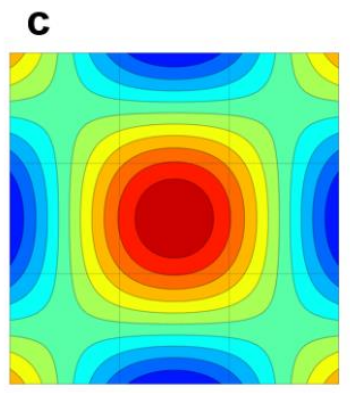

$j=2$

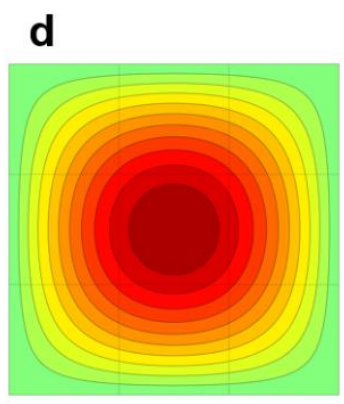

$j=3$

Figure S2. 2-D contour plots of the proposed phase representation including additional bases. Black grid lines denote borders between meta-atoms. (a) A harmonic basis whose periodicity is $2 \Lambda$. (b) and (c) are repeated from Figure 2a for comparison. (d) A harmonic with $6 \Lambda$ periodicity. The component does not contribute much to high-contrast patterns because it is close to plane wave that forms a periodic response.

We elaborate on the choice of $n_{h}=2(j=1$ or $j=2)$ in Eq. (1). Three primary considerations are as follow:

- Experimental reproduction of optimized phase distributions by SLMs imposes a constraint on the resolution. Based on our setting in Eq. (1), $j=1$ involves the minimum feature resolution that SLMs of usual specification can generate (Figure S2b). Finer phase distributions $(j<1)$ were not addressed as they cannot be produced in experiments (Figure S2a).

- Plane wave contributes to periodic response. This implies that harmonic components with large periods (e.g., $j=3$ in Figure S2d) hardly contribute to high-contrast localization patterns that this study aims for. Based on our numerical experiments, the components with $j>2$ have insignificant influence on aperiodic responses.

- Including higher-order harmonics provides more design freedom in incident phase. As a trade-off, however, it increases the dimensionality of design space to be searched during the phase optimization.

Based upon all the considerations, we used the proposed phase representation $(j=1$ or $j=2)$ with six design variables $\mathbf{X}=\left[\varphi_{1}, \alpha_{1}, \beta_{1}, \varphi_{2}, \alpha_{2}, \beta_{2}\right]^{\mathrm{T}}$ for our phase representation.

\section{S3. Bayesian Optimization}

Bayesian optimization (BO) is an efficient global optimization algorithm that builds on a probabilistic metamodel. A key distinction of $\mathrm{BO}$ is that it exploits all the data available for sequential model update and inference. BO starts with a surrogate and progressively refines it using new draws determined by an infill criterion. Two main pillars of BO are (i) a prior on a black-box function of interest and (ii) an acquisition function.

The prior encodes beliefs about the function to be optimized. Gaussian process (GP) priors have been widely used, mainly due to the computational advantage on estimating conditionals and marginals. ${ }^{2} \mathrm{~A}$ GP is a collection of random variables, any subset of which are jointly Gaussian. ${ }^{3} y(\mathbf{x})$ is interpreted as a realization of the random process. The GP model is fully specified by mean and covariance functions as follows:

$$
y(\boldsymbol{x})=\mu+\xi(\boldsymbol{x}),
$$


where $\mathbf{x}$ represents $D$-dimensional design variables $\mathbf{x}=\left(x_{1}, \cdots, x_{D}\right) ; \mu$ is a constant mean prior; $\xi$ denotes a zeromean GP whose covariance function $\operatorname{cov}(\cdot, \cdot)$ reads:

$$
\operatorname{cov}\left(\boldsymbol{x}, \boldsymbol{x}^{\prime}\right)=\sigma^{2} r\left(\boldsymbol{x}, \boldsymbol{x}^{\prime}\right) \text { and } r\left(\boldsymbol{x}, \boldsymbol{x}^{\prime}\right)=\exp \left(-\frac{1}{2} \sum_{d=1}^{D}\left(\frac{x_{d}-x_{d^{\prime}}}{\theta_{d}}\right)^{2}\right),
$$

where $\sigma^{2}$ is the process variance; $r(\cdot, \cdot)$ is the Gaussian (a.k.a. squared exponential) correlation, which depends on both scale parameters $\left\{\theta_{1}, \cdots, \theta_{D}\right\}$ and an Euclidean distance between a pair of inputs. The covariance kernel serves as the core component of GP regression because it reflects a prior on functions (e.g., stationarity) and measures similarity between data. ${ }^{4}$ Given a dataset and prior, fitting a GP model boils down to identifying an optimal point estimate of hyperparameters $\boldsymbol{\theta}=\left\{\mu, \sigma^{2} ; \theta_{1}, \cdots, \theta_{D}\right\}$. This usually involves maximum likelihood estimation by numerical optimization.

Meanwhile, an acquisition function determines where to sample next by forming a utility function of samples available. It takes both exploitation (function min/maximization) and exploration (uncertainty reduction) into account. We employ the expected improvement (EI) criterion ${ }^{5}$ that predicts the improvement of unseen samples over the current best. Assuming a GP prior, EI for a maximization problem has the closed form expression as ${ }^{6}$

$$
\begin{gathered}
E I(\boldsymbol{x}) \equiv \mathbb{E}\left[\max \left(0, y(\boldsymbol{x})-f^{*}\right)\right]=s(\boldsymbol{x})[u \Phi(u)+\phi(u)] \\
\text { where } u=\frac{y(\boldsymbol{x})-f^{*}}{s(\boldsymbol{x})}, \Phi(u)=\frac{1}{2} \operatorname{erf}\left(\frac{u}{\sqrt{2}}\right)+\frac{1}{2} \text {, and } \phi(u)=\frac{1}{\sqrt{2}} \exp \left(-\frac{u^{2}}{2}\right) \text {. }
\end{gathered}
$$

$f^{*}$ is the current best at a given iteration; $s^{2}(\mathbf{x})$ is the variance of model prediction $\hat{y}(\boldsymbol{x}) ; \Phi(u)$ and $\phi(u)$ are the normal cumulative distribution and normal probability distribution, respectively. EI serves as an effective acquisition function for deterministic problems. Noisy responses, on the other hand, can be better addressed by other acquisition functions. Readers can refer to Ref. ${ }^{7}$ for relevant guidelines.

Now that we have chosen a probabilistic metamodel (GP) and an infill criterion (EI), BO can be performed. As an inspectional search of design space, space-filing design is widely used to generate an initial dataset $\mathcal{D}_{0}$. Our choice is Latin hypercube sampling of $10 \times D=60$ observations where $D=6$ is the dimension of design variables. The main loop at iteration $i$ starts by fitting a GP on the full dataset $\mathcal{D}_{i}$. Using the GP posterior, EI(x) predicts the expected utility of unseen points. $\operatorname{argmax}_{\mathbf{x}} E I(\mathbf{x})$ becomes the new sampling location $\mathbf{x}_{i+1}$ to be evaluated by simulations (full-wave analysis in this work). The new input-output pair $\left(\mathbf{x}_{i+1}, y_{i+1}\right)$ augments the database as $\mathcal{D}_{i+1}=\left\{\mathcal{D}_{i},\left(\mathbf{x}_{i+1}, y_{i+1}\right)\right\}$ and a next iteration starts with updating the GP model. 


\section{S4. A schematic of wave analysis}

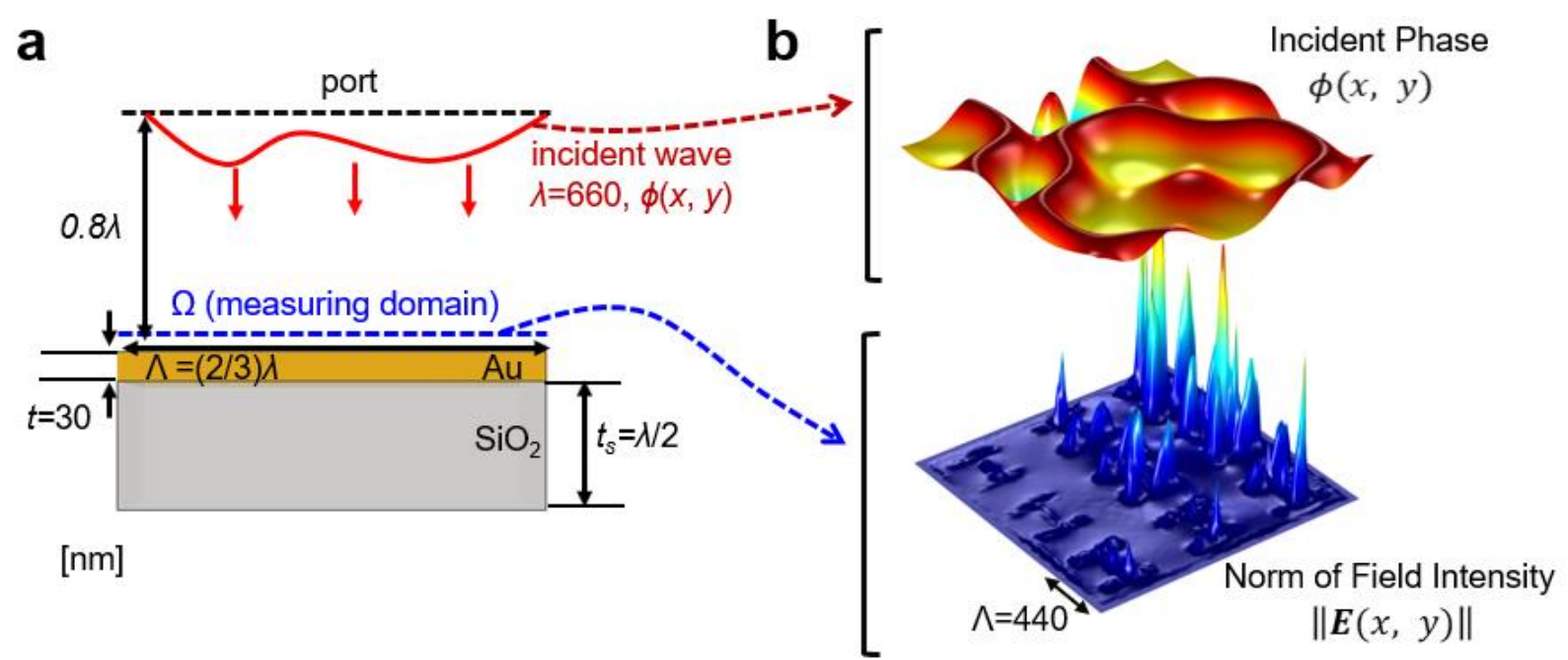

Figure S3. A schematic of wave analysis. (a) A side view of the whole plasmonic metasurface system. (b) Virtual profiles of a modulate incident phase and the resulting norm of electric field intensity.

\section{Reference}

(1) Voelz, D. G. Computational Fourier Optics: A MATLAB Tutorial. SPIE press Bellingham. 2011.

(2) Snoek, J.; Larochelle, H.; Adams, R. P. Practical Bayesian Optimization of Machine Learning Algorithms. Adv. Neural Inf. Process. Syst. 2012, 4, 2951-2959.

(3) Rasmussen, C. E. Gaussian Processes in Machine Learning. Lect. Notes Comput. Sci. (including Subser. Lect. Notes Artif. Intell. Lect. Notes Bioinformatics) 2004, 3176, 63-71.

(4) Eberhard, J.; Geissbuhler, V. [Book] Gaussian Processes for Machine Learning; 2000; Vol. 7.

(5) Jones, D. R.; Schonlau, M.; Welch, W. J. Efficient Global Optimization of Expensive Black-Box Functions. J. Glob. Optim. 1998, 13 (4), 455-492.

(6) Frean, M.; Boyle, P. Using Gaussian Processes to Optimize Expensive Functions. In AI 2008: Advances in Artificial Intelligence; Wobcke, W., Zhang, M., Eds.; Springer Berlin Heidelberg: Berlin, Heidelberg, 2008; pp 258-267.

(7) Picheny, V.; Wagner, T.; Ginsbourger, D. A Benchmark of Kriging-Based Infill Criteria for Noisy Optimization. Struct. Multidiscip. Optim. 2013, 48 (3), 607-626. 